\title{
Implementing the SEA application for organic chemistry: arrow-pushing versus electron-moving techniques
}

\begin{abstract}
The models used in organic chemistry are usually rigid; it is difficult for students to rapidly transfer between the macroscopic, submicroscopic, and symbolic levels of thought. As a result, students' perceptions of these models are sometimes incorrect. In organic synthesis students should comprehend information about reactions and, simultaneously, visualize the inverse of these reactions. Without deep understanding of the mechanistic aspects of organic reactions, students will face problems in an advanced chemistry course. Another problem in teaching organic chemistry effectively is the wide range of abilities and academic interests of students. The introductory organic chemistry class caters for students from various science and technology fields. Based on this context, a computer application was used as an alternative approach for teaching and learning organic chemistry with the assumption that animations could assist students to better understand concepts. In a study by Gilbert, Reiner and Nakhleh (2008), it was implied that animated presentations could exert an extra cognitive load on students. Consequently, a relevant question that educators need to answer is ñHow visuals should be animated in science instruction?ò This study examined how an animation based computer application that applied an electron-moving technique was used as a supplementary material in a first year Organic Chemistry course and its effect on the performance of students. Using pre and post-test design, the study involved a comparison between an experimental group of first year students $(n=28)$ who followed instructions that used moving-electron technique in a blended mode and a conventional group of first year students $(n=27)$ who used arrow-pushing technique to learn the fundamental concept of organic reaction mechanism. The finding suggest the potential of using electron-moving technique as an instructional approach based on the improved post-test performance as compared to arrow-pushing techniques.
\end{abstract}

Keyword: Organic chemistry; Animation; Arrow pushing technique; Electron moving technique 